PHYSICAL REVIEW D 93, 095028 (2016)

\title{
Supersoft supersymmetry, conformal sequestering, and single scale supersymmetry breaking
}

\author{
Ran Ding, ${ }^{1}$ Tianjun $\mathrm{Li}^{2,3}$ Florian Staub, ${ }^{4}$ and Bin $\mathrm{Zhu}^{5}$ \\ ${ }^{1}$ Center for High-Energy Physics, Peking University, Beijing 100871, People's Republic of China \\ ${ }^{2}$ State Key Laboratory of Theoretical Physics and Kavli Institute for Theoretical Physics, China (KITPC), \\ Institute of Theoretical Physics, Chinese Academy of Sciences, Beijing 100190, People's Republic of China \\ ${ }^{3}$ School of Physical Electronics, University of Electronic Science and Technology of China, \\ Chengdu 610054, People's Republic of China \\ ${ }^{4}$ Theory Division, CERN, 1211 Geneva 23, Switzerland \\ ${ }^{5}$ Institute of Physics Chinese Academy of Sciences, Beijing 100190, People's Republic of China
}

(Received 7 October 2015; published 31 May 2016)

\begin{abstract}
Supersymmetric standard models (SSMs) with Dirac gauginos have the appealing supersoft property that they only cause finite contributions to scalar masses. Considering gauge mediated SUSY breaking with conformal sequestering and assuming there is one and only one fundamental parameter with dimension mass arising from supersymmetry breaking, we find a cancellation between the dominant terms that contribute to the electroweak fine tuning (EWFT). The resulting EWFT measure can be of order one even for supersymmetric particle masses and $\mu$-terms in the TeV range.
\end{abstract}

DOI: 10.1103/PhysRevD.93.095028

\section{INTRODUCTION}

Supersymmetry (SUSY) provides a natural solution to the gauge hierarchy problem in the standard model (SM). In the supersymmetric SMs (SSMs) with $R$ parity, gauge coupling unification can be achieved, and the lightest supersymmetric particle (LSP) is a dark matter candidate. However, after the first run of the Large Hadron Collider (LHC), the former top candidate for physics beyond the SM, the minimal SSM (MSSM), has lost a lot of its attraction. One reason is the discovery of the SM-like Higgs boson with a mass of $125 \mathrm{GeV}[1,2]$. In order to obtain the correct Higgs mass, there are two possibilities in the MSSM: either there must be a very large mixing among the supersymmetric partners of top quarks, or the SUSY breaking soft masses must be much heavier than naively expected. The first possibility is often disfavored by charge and color breaking minima [3-7], while the second one raises the question if the MSSM is really a natural solution to gauge hierarchy problem. This has caused an increasing interest in nonminimal SUSY models. The focus was mainly on models which enhance the Higgs at tree level to reduce the fine-tuning (FT) [8-19]. In addition, the other ideas like $R$-symmetric SSMs with Dirac instead of Majorana gauginos became much more popular in the last few years [20-55]. On the one hand, such models are known to be supersoft since they only give finite contributions to scalar masses $[20,34]$. On the other hand, they

Published by the American Physical Society under the terms of the Creative Commons Attribution 3.0 License. Further distribution of this work must maintain attribution to the author(s) and the published article's title, journal citation, and DOI. can reduce existing mass limits from SUSY searches and weaken bounds from flavor physics [24]. It is somehow surprising, but the electroweak fine tuning (EWFT) question in the SSMs with Dirac gauginos and a specific SUSY breaking mechanism has not been addressed so far. So we shall close this gap here.

The single scale SUSY provides an elegant solution to the SUSY EWFT problem [56-58]. In particular, the original conditions for string inspired SSMs are mainly [58]: (1) The Kähler potential and superpotential can be calculated in principle or at least inspired from a fundamental theory such as string theory with suitable compactifications; (2) There is one and only one chiral superfield which breaks supersymmetry; (3) All the mass parameters in the SSMs must arise from supersymmetry breaking. With these conditions, one can show that the SUSY EWFT measure is automatically of order one. The above conditions seem to be too strong, thus, we point out that the essential condition is: there is one and only one fundamental mass parameter and the coefficients to set the different mass scales to be determined. Or simply speaking, all dimensionful parameters in the SSMs are correlated. In particular, all dimensionful parameters can be further relaxed to all the dimensional parameters with large EWFT measures, and we shall call it the effective single scale condition.

In the minimal $R$-symmetric SSM (MRSSM) with Dirac gauginos, we present for the first time the SUSY breaking soft terms from gauge mediated SUSY breaking (GMSB) [28] with conformal sequestering [59-61], and find that the naive EWFT measure turns out to be similar to the other SSMs, except the minor improvements due to supersoft property and additional loop contributions to the Higgs 
boson mass. With our above updated condition for single scale SUSY, we show a perfect cancellation analytically and numerically between the dominant terms that contribute to the EWFT. The resulting EWFT measure can be of order one even for the supersymmetric particle (sparticle) masses in the $\mathrm{TeV}$ range. In particular, it is not necessary that the dimensionful parameters in the superpotential have to be tuned to be small as this is usually the case. In a wide range of the parameter space we find a precise cancellation among different contributions to the EWFT measures.

\section{THE SUSY BREAKING SOFT TERMS}

The generic new soft terms in the MRSSM are

$$
\mathcal{L}=\left(m_{D} \lambda_{i} \psi_{A_{i}}+b_{A} A^{2}+\text { H.c. }\right)+m_{A}^{2}|A|^{2},
$$

where $\lambda$ is a gaugino, $\psi$ and $A$ are the fermionic and scalar components of a chiral adjoint superfield, $m_{D}$ is the Dirac gaugino mass, and $b_{A}$ and $m_{A}^{2}$ are the holomorphic and, respectively, nonholomorphic masses.

In the simplest ansatz that the origin of the Dirac mass term is the operator for gauge field strengths $W_{\alpha}^{\prime}$ and $W_{j}^{\alpha}$

$$
W_{\text {ssoft }}=\frac{W_{\alpha}^{\prime} W_{j}^{\alpha} A_{j}}{\Lambda},
$$

a massless scalar in the adjoint representation is predicted [41]. This observation has triggered efforts in constructing phenomenological reliable models with Dirac gauginos $[47,62,63]$. In general, the aim is to get $m_{D}^{2} \sim m_{A}^{2} \sim b_{A}$. However, if $b_{A}$ and $m_{D}$ are generated at one loop, $b_{A}$ is naturally larger than $m_{D}^{2}$ by a loop factor of $16 \pi^{2}$. To address this $m_{D}-b_{A}$ problem and generate the proper Dirac gaugino and scalar masses, we introduce two pairs of messenger fields for the gauge mediated supersymmetry breaking (GMSB) [28] and consider the conformal sequestering [59-61]. Supposing the hidden sector interactions are strong below the messenger scale $M_{\text {mess }}$ down to some scale where conformality is broken, we obtain

$$
\begin{aligned}
m_{D_{i}} & =\frac{g_{i}}{16 \pi^{2}} \frac{C_{D_{i}} \lambda_{i}}{6 \sqrt{2}} \frac{\Lambda_{F}^{\prime 2}}{M_{\text {mess }}}, \\
b_{A_{i}} & =-\frac{1}{16 \pi^{2}} \frac{C_{b_{i}} \lambda_{i}^{2}}{2^{\delta_{i}}} \Lambda_{F}^{\prime 2}, \\
m_{A_{i}}^{2} & =\left(\frac{1}{32 \pi^{2}} \frac{\lambda_{i}^{2}}{2^{\delta_{i}}}+\frac{1}{128 \pi^{4}} \sum_{i} C_{i}\left(A_{i}\right) g_{i}^{4}\right) C_{A_{i}} \Lambda_{F}^{\prime 2}, \\
m_{\phi}^{2} & =\frac{1}{128 \pi^{4}} \sum_{i} C_{i}(\phi) g_{i}^{4} C_{\phi} \Lambda_{F}^{\prime 2},
\end{aligned}
$$

where $\left(\delta_{1}, \delta_{2}, \delta_{3}\right)=(0,1,1), g_{i}$ and $\lambda_{i}$ are gauge and Yukawa couplings, $\phi$ represents scalars not appearing in the adjoint representation, $C_{D_{i} / b_{i} / A_{i} / \phi}$ is the conformal sequestering suppression factor, and $C_{i}\left(A_{i} / \phi\right)$ is the quadratic Casimir index. For simplicity, we assume $C_{b_{i}}=C_{A_{i}}=C_{\phi} \equiv C_{X X}$, and define

$$
\begin{aligned}
y_{i} & \equiv \frac{C_{D_{i}} \lambda_{i}}{6 \sqrt{2}} \frac{\Lambda_{F}^{\prime 2}}{M_{\text {mess }} \Lambda_{D}}, \\
\Lambda_{F}^{2} & \equiv C_{X X} \Lambda_{F}^{\prime 2},
\end{aligned}
$$

where $\Lambda_{D}$ and $\Lambda_{F}$ are roughly the same mass scales. Assuming that $C_{X X} \ll C_{D_{i}}$ and $10 \lambda_{i} \leq g_{1}^{2} / 2 \sqrt{2} \pi$, we approximately have

$$
\begin{gathered}
m_{D_{i}}=\frac{g_{i} y_{i}}{16 \pi^{2}} \Lambda_{D}, \quad b_{A_{i}} \simeq 0, \\
m_{A_{i} / \phi}^{2} \simeq \frac{1}{128 \pi^{4}} \sum_{i} C_{i}\left(A_{i} / \phi\right) g_{i}^{4} \Lambda_{F}^{2} .
\end{gathered}
$$

\section{THE MRSSM}

The particle content of the MRSSM is the MSSM extended by adjoint superfields for all gauge groups necessary to construct Dirac gaugino masses as well as by two chiral isodoublets $R_{u}$ and $R_{d}$ with $R$ charge 2 to build $\mu$ like terms. Thus, the superpotential is

$$
\begin{aligned}
W= & -Y_{d} \hat{d} \hat{q} \hat{H}_{d}-Y_{e} \hat{e} \hat{l} \hat{H}_{d}+Y_{u} \hat{u} \hat{q} \hat{H}_{u}+\mu_{D} \hat{R}_{d} \hat{H}_{d} \\
& +\mu_{U} \hat{R}_{u} \hat{H}_{u}+\hat{S}\left(\lambda_{d} \hat{R}_{d} \hat{H}_{d}+\lambda_{u} \hat{R}_{u} \hat{H}_{u}\right) \\
& +\lambda_{d}^{T} \hat{R}_{d} \hat{T} \hat{H}_{d}+\lambda_{u}^{T} \hat{R}_{u} \hat{T} \hat{H}_{u} .
\end{aligned}
$$

All the other terms are forbidden by the $R$-symmetry as the Majorana gaugino masses and trilinear soft-breaking couplings are. However, a soft-breaking term $B_{\mu}$ necessary to give mass to the pseudoscalar Higgs is allowed by this symmetry. The tree-level Higgs mass is even smaller than the MSSM because of negative contributions from the new $D$-terms proportional to the Dirac gaugino masses. Moreover, the stops cannot be used to push this mass significantly since all $A$-terms are forbidden by $R$-symmetry. Nevertheless, it has been shown that the large loop corrections stemming from the new superpotential terms $\lambda_{i}$ and $\lambda_{i}^{T}(i=u, d)$ increase the Higgs mass to the demanded level $[64,65]$. Moreover, this model is consistent with gauge coupling unification [49]. Thus, it is natural to embed it in a constrained SUSY breaking scenario.

We use the boundary conditions defined in Eqs. (5)-(6) in the limit $\Lambda_{D} / M \rightarrow 0$ to calculate most soft masses at the conformal scale $M$. Only the soft-mass for the singlet $m_{s}^{2}$ and $B_{\mu}$, which can also be generated via Yukawa mediations, are derived from the minimization conditions at the vacuum. The other two minimization conditions are used to calculate $\mu_{D}$ and $\mu_{U}$. In short, we have the following input parameters

$$
\Lambda_{F}, \Lambda_{D}, M, y_{i}, \lambda_{u}, \lambda_{d}, \lambda_{u}^{T}, \lambda_{d}^{T}, \tan \beta, v_{s}, v_{T},
$$


where $\tan \beta \equiv\left\langle H_{u}^{0}\right\rangle /\left\langle H_{d}^{0}\right\rangle$, and $v_{s}$ and $v_{T}$ are the vacuum expectation values (VEVs) of the singlet and neutral triplet. Also, we assume $\mu_{D}$ and $\mu_{U}$ are positive.

\section{NATURALNESS}

To quantize the EWFT size, we adopt the measure introduced in Refs. [66,67]

$$
\Delta_{F T} \equiv \operatorname{Max}\left\{\Delta_{\alpha}\right\}, \quad \Delta_{\alpha} \equiv\left|\frac{\partial \ln M_{Z}^{2}}{\partial \ln \alpha}\right|,
$$

where $\alpha$ is a set of independent parameters, and $\Delta_{\alpha}^{-1}$ gives an estimate of the accuracy to which the parameter $\alpha$ must be tuned to get the correct electroweak symmetry breaking (EWSB) scale [68]. The smaller $\Delta_{F T}$, the more natural the model under consideration is. We use the conformal scale $M$ as a reference scale and calculate the FT with respect to $\left\{\Lambda_{F}, \Lambda_{D}, y_{i}, \lambda_{d, u}, \lambda_{d, u}^{T}, \mu_{D, U}, m_{s}^{2}, B_{\mu}\right\}$.

For large regions in parameter space, the main EWFT sources are $\mu_{U}$ and the scale $\Lambda_{F}$ because of the impact on the running soft mass $m_{H_{u}}^{2}$ responsible for EWSB. If we only include the terms proportional to top Yukawa coupling in the running, we can estimate the EWFT measures for these two parameters to be

$$
\left.\Delta_{F T}\left(\Lambda_{F}\right) \approx \mid \frac{\sqrt{2} \Lambda_{F}^{2}}{384 \pi^{4} v^{2}}\left(\left(32(-1+R)+9(1+R) g_{2}^{4}\right)\right)\right) \mid,
$$

$$
\Delta_{F T}\left(\mu_{U}\right) \approx\left|R \cdot 4 \sqrt{2} \frac{\mu_{U}^{2}(M)}{v^{2}}\right|,
$$

where $R=e^{\left(\left(3 \log \left(M_{\mathrm{SUSY}} / M\right) Y_{t}^{2}\right) /\left(16 \pi^{2}\right)\right)}$, and $\mu_{U}(M)$ is the running value of $\mu_{U}$ at the conformal scale. For simplicity we assumed that $Y_{t}$ does not change significantly between the SUSY breaking and conformal scales, but our conclusion is independent of this approximation. As usual, one finds that the FT measure increases quickly with increasing values for the SUSY breaking scale and/or the scale of the dimensionful parameters in the superpotential. For $\mu_{U}$ in the $\mathrm{TeV}$ range, it seems not to be possible to find a FT measure below 100 unless the conformal scale is very low.

Assuming that all the parameters with dimension mass are correlated at the conformal scale, which is defined as single scale supersymmetry, we have

$$
\Lambda_{D} \sim \Lambda_{F} \sim \mu_{D} \sim \mu_{U} \sim m_{s} \sim \sqrt{B_{\mu}} .
$$

The underlying assumption is: there is one and only one fundamental parameter with dimension mass and the coefficients to set the different scales are calculable. However, a concrete construction of such a model is beyond the scope of this paper. As we will show, the single scale SUSY condition can be relaxed further to the effective conformal sequestering single scale SUSY condition, and for the following discussion only $\Lambda_{F} \sim \mu_{U}$ is necessary since their corresponding EWFT measures are relatively large while all the rest are small and negligible.

To study the effect of this correlation, we first determine $\mu_{U}(M)$ from the tadpole equations in the limit $v_{T} \rightarrow 0$ and $\lambda_{u} \rightarrow 0$. We obtain

$$
\begin{aligned}
\mu_{U}(M)= & \frac{1}{96 \pi^{2}\left(\lambda_{d}^{T, 2}-\lambda_{u}^{T, 2} \tan ^{2} \beta\right)}\left(6 g_{2}^{2} \lambda_{u}^{T} \tilde{\Lambda}_{D} \tan ^{2} \beta\right. \\
& \left.+\sqrt{3 \lambda_{d}^{T, 2} \tan ^{2} \beta\left(12 g_{2}^{4} \tilde{\Lambda}_{D}^{2}+\lambda_{u}^{T, 2} \Lambda_{F}^{2} R\right)-3 \lambda_{d}^{T, 4} \Lambda_{F}^{2} R}\right),
\end{aligned}
$$

where $\tilde{\Lambda}_{D} \equiv y_{2} \Lambda_{D}$. If we combine Eqs. (10) and (11), we get the correlated FT measure

$$
\Delta_{F T}^{C}=\frac{\sqrt{2} \lambda_{u}^{T, 2} \Lambda_{F}^{2} \tan ^{2} \beta R}{384 \pi^{4} v^{2}\left(\lambda_{d}^{T, 2}-\lambda_{u}^{T, 2} \tan ^{2} \beta\right)}+\frac{\tilde{\Lambda}_{D}}{\Lambda_{F}} F_{1}+\frac{\tilde{\Lambda}_{D}^{2}}{\Lambda_{F}^{2}} F_{2},
$$

where $F_{1}$ and $F_{2}$ are functions of $g_{2}, \lambda_{i}^{T}$ and $\tan \beta$ which we skip for brevity. The last two terms can be suppressed in the limit $\tilde{\Lambda}_{D} \ll \Lambda_{F}$. This is also the preferred limit, because large $\tilde{\Lambda}_{D}$ would cause large wino masses which reduce the tree-level Higgs mass. The first term becomes very small for $\lambda_{u}^{T} \rightarrow 0$. We have checked numerically that these estimates reproduce the correct behavior to a large extent even if we include the correlation to all other dimensionful parameters. For this purpose, we implement the model in the Mathematica package SARAH [69-73] and generate FORTRAN code for SPHENO [74,75] to calculate the FT measures using the full two-loop renormalization group equations (RGEs) based on Ref. [37]. The calculated values for $\Delta_{F T}\left(\Lambda_{F}\right), \Delta_{F T}\left(\mu_{U}\right)$ and $\Delta_{F T}^{C}$ as function of $\Lambda_{D}, \lambda_{d}^{T}$, and $\lambda_{u}^{T}$ are shown in Fig. 1. We find that $\Delta_{F T}^{C}$ tends to be very small for small $\Lambda_{F}$ and $\lambda_{u}^{T}$ together with large $\lambda_{d}^{T}$, while $\Delta_{F T}\left(\Lambda_{F}\right)$ and $\Delta_{F T}\left(\mu_{U}\right)$ are several orders larger.

Our proposal is completely different from focus point SUSY often considered in the MSSM [76,77]: in the focus point SUSY $m_{H_{u}}^{2}$ is rather insensitive to the UV parameters because of specific hierarchies in the corresponding $\beta$-function. While this suppresses the FT with respect to $m_{H_{u}}^{2}$ one has always to tune $\mu$ to be small in order to obtain a low overall FT. In our proposal, there is no need that the FT with respect to the $\mu$-term is small nor the cancellations in the running of $m_{H_{u}}^{2}$ are needed because there is a precise cancellation among these two sources.

We have checked whether this mechanism can be applied to the MSSM with the minimal GMSB. And indeed, we have found there a good cancellation for large $\tan \beta$ if we relate the SUSY breaking scale $\Lambda$ and $\mu$ at the messenger scale. However, this cancellation in the MSSM is not as good as the MRSSM considered here. The point is that the contributions from the Majorana gaugino masses to the running of $m_{H_{u}}^{2}$ are absent in the MRSSM. The $\Delta_{F T}^{C}$ in 

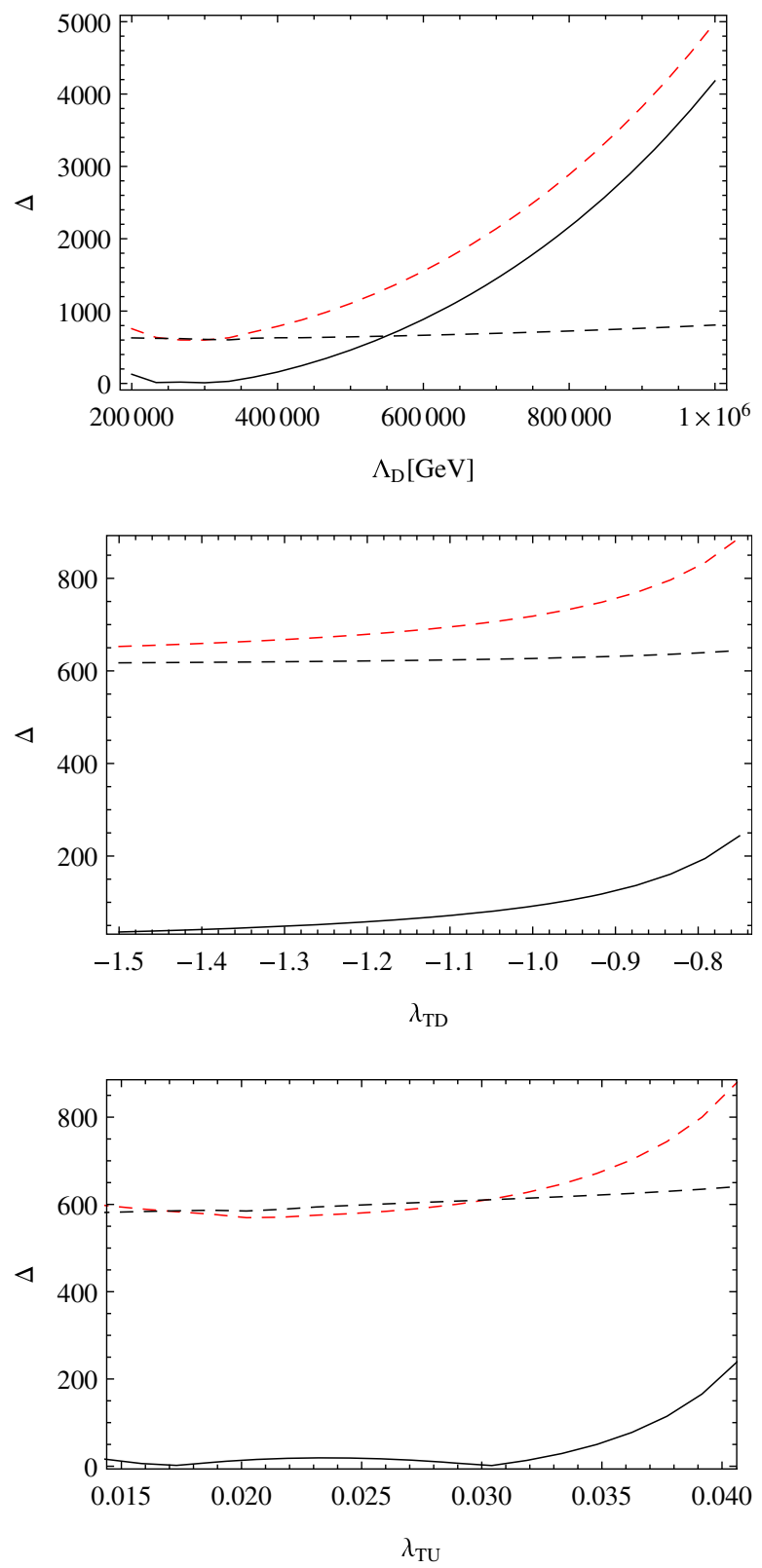

FIG. 1. The calculated FT measures $\Delta_{F T}\left(\mu_{U}\right)$ (dashed red), $\Delta_{F T}\left(\Lambda_{F}\right)$ (dashed black), $\Delta_{F T}^{C}$ (full black line) as function of $\Lambda_{D}$ (first row), $\lambda_{d}^{T}$ (second row) and $\lambda_{u}^{T}$ (third row). The other parameters were set to $\Lambda_{F}=2.7 \times 10^{5} \mathrm{GeV}, \Lambda_{D}=$ $2.0 \times 10^{5} \mathrm{GeV}, \quad M=10^{12} \mathrm{GeV}, \quad \tan \beta=20, \quad y_{i}=(0.6,-0.15$, $-0.75), \lambda_{s, d}=(-0.78,-0.01), \lambda_{s, d}^{T}=(-1,0.037), v_{s}=-5 \mathrm{GeV}$, $v_{T}=-0.25 \mathrm{GeV}$.

the MSSM is always bigger than the MRSSM, but still very good and well below 100. We test it numerically by removing the gaugino contribution terms "by hand" from the $\beta$-functions of scalars, and indeed we can recover a similar cancellation as described here and $\Delta_{F T}^{C}$ drops to very small values. Thus, the supersoft character of Dirac gauginos together with an underlying correlation among dimensionful parameters results in a very natural model. The detailed study for the MSSM will be given elsewhere.

\section{BENCHMARK SCENARIOS}

In the EWFT discussion so far, we have neglected all other current experimental constraints that must be fulfilled. In particular, the mass limits on the SUSY particles from direct and indirect searches as well as the measurement of the SM-like Higgs mass exclude large parameter regions in SUSY models today. We can use the generated SPHENO version to check all these constraints. It is especially worthwhile to point out that the Higgs mass is also calculated at the two-loop level including all model specific contributions in the gaugeless limit [78,79]. Therefore, the theoretical uncertainty is of the same level as in the MSSM and can be estimated to be $O(3 \mathrm{GeV})$. We show the input and the most important output parameters for two benchmark scenarios in Table I.

One sees that the uncorrelated EWFT measures in our model are already smaller than in the usual MSSM with GMSB. The reason are the additional loop corrections which weak the need for very heavy stops significantly. For example, we have very large $\lambda_{d}$ couplings for BP2. This is similar to the MSSM extensions with vectorlike (s)tops where the additional loop corrections cause a significant improvement in the EWFT measure [80]. Also the two-loop

TABLE I. The input parameters, important particle spectra, and EWFT measures for two benchmark scenarios. $\operatorname{Max}\left(\Delta_{F T}(\lambda)\right)$ is the maximal EWFT measure for $\lambda_{d, u}$ and $\lambda_{d, u}^{T}$.

\begin{tabular}{|c|c|c|}
\hline & BP1 & BP2 \\
\hline \multicolumn{3}{|c|}{ Input } \\
\hline$\Lambda_{F}\left[10^{5} \mathrm{GeV}\right]$ & 2.7 & 2.0 \\
\hline$\Lambda_{D}\left[10^{5} \mathrm{GeV}\right]$ & 2.0 & 2.2 \\
\hline$M\left[10^{7} \mathrm{GeV}\right]$ & 1.0 & 1.0 \\
\hline$y_{i}$ & $-(-0.63,0.15,0.75)$ & $-(0.45,0.16,1.1)$ \\
\hline$\lambda_{d, u}$ & $-(0.78,0.01)$ & $(-1.45,0.09)$ \\
\hline$\lambda_{d, u}^{T}$ & $(-1.0,0.037)$ & $(-1.60,0.07)$ \\
\hline $\tan \beta$ & 20 & 20 \\
\hline$v_{s, T}[\mathrm{GeV}]$ & $-(5.0,0.25)$ & $-(4.0,0.56)$ \\
\hline \multicolumn{3}{|c|}{ Output } \\
\hline \multicolumn{3}{|l|}{$\mu_{U}[\mathrm{GeV}]$} \\
\hline \multicolumn{3}{|c|}{ Masses } \\
\hline$m_{h}[\mathrm{GeV}]$ & 123.5 & 122.4 \\
\hline$m_{\tilde{g}}[\mathrm{GeV}]$ & 1620.5 & 2316.9 \\
\hline$m_{\tilde{q}}[\mathrm{GeV}]$ & $\sim 3000$ & $\sim 2300$ \\
\hline$m_{\tilde{l}_{R}}[\mathrm{GeV}]$ & $\sim 500$ & $\sim 400$ \\
\hline$m_{\tilde{l}_{l}}[\mathrm{GeV}]$ & $\sim 1000$ & $\sim 1000$ \\
\hline$m_{\tilde{\chi}^{0}}^{l_{L}}[\mathrm{GeV}]$ & 151.2 & 159.0 \\
\hline \multicolumn{3}{|c|}{$\Delta_{F T}$} \\
\hline $\operatorname{Max}\left(\Delta_{F T}(\lambda)\right)$ & 0.7 & 1.8 \\
\hline$\Delta_{F T}\left(\Lambda_{F}\right)$ & 342.5 & 180.0 \\
\hline$\Delta_{F T}\left(\Lambda_{D}\right)$ & 0.2 & 0.1 \\
\hline$\Delta_{F T}\left(\mu_{U}\right)$ & 342.8 & 186.7 \\
\hline$\Delta_{F T}\left(\mu_{D}\right)$ & 4.2 & 9.2 \\
\hline$\Delta_{F T}\left(B_{\mu}\right)$ & $\begin{array}{l}4.3 \\
\Delta_{F T}^{C}\end{array}$ & 9.1 \\
\hline$\Delta_{F T}^{C}$ & $0.2^{F T}$ & 6.8 \\
\hline
\end{tabular}


corrections are enhanced due to the presence of scalar octets. Moreover, the correlated EWFT becomes much smaller due to the precise cancellation between the contributions from $\Lambda_{F}$ and $\mu_{U}$. For BP1 the resulting EWFT is even smaller for the dimensionless parameters. Because of the slightly larger value of $\lambda_{u}^{T}$, as expected, the cancellation for BP2 is not working as good as for $\mathrm{BP} 1$, although $\Delta_{F T}^{C}$ is still very small.

\section{CONCLUSION}

We considered the GMSB with conformal sequestering, and found that the naive EWFT measures in the MRSSM are similar to the other SSMs except the minor improvements due to supersoft property and additional loop contributions to the Higgs boson mass. With the effective single scale SUSY condition that all dimensionful parameters with large EWFT measures are correlated, we showed explicitly an excellent cancellation between the dominant terms that contribute to the EWFT. As we expected, the correlated EWFT measure is of unit order even for the $\mathrm{TeV}$-scale supersymmetric particle masses.

\section{ACKNOWLEDGMENTS}

We thank Jessica Goodman for very useful discussions. This research was supported in part by the Natural Science Foundation of China under Grants No. 11135003, No. 11275246, and No. 11475238 (TL).
[1] S. Chatrchyan et al. (CMS Collaboration), Phys. Lett. B 716, 30 (2012).

[2] G. Aad et al. (ATLAS Collaboration), Phys. Lett. B 716, 1 (2012).

[3] J. Camargo-Molina, B. O'Leary, W. Porod, and F. Staub, J. High Energy Phys. 12 (2013) 103.

[4] N. Blinov and D. E. Morrissey, J. High Energy Phys. 03 (2014) 106.

[5] D. Chowdhury, R. M. Godbole, K. A. Mohan, and S. K. Vempati, J. High Energy Phys. 02 (2014) 110.

[6] J. E. Camargo-Molina, B. Garbrecht, B. O'Leary, W. Porod, and F. Staub, Phys. Lett. B 737, 156 (2014).

[7] U. Chattopadhyay and A. Dey, J. High Energy Phys. 11 (2014) 161.

[8] M. Bastero-Gil, C. Hugonie, S. King, D. Roy, and S. Vempati, Phys. Lett. B 489, 359 (2000).

[9] R. Dermisek and J. F. Gunion, Phys. Rev. D 73, 111701 (2006).

[10] Y. Zhang, H. An, X.-d. Ji, and R. N. Mohapatra, Phys. Rev. D 78, 011302 (2008).

[11] U. Ellwanger, G. Espitalier-Noel, and C. Hugonie, J. High Energy Phys. 09 (2011) 105.

[12] G. G. Ross and K. Schmidt-Hoberg, Nucl. Phys. B862, 710 (2012).

[13] M. Hirsch, M. Malinsky, W. Porod, L. Reichert, and F. Staub, J. High Energy Phys. 02 (2012) 084.

[14] G. G. Ross, K. Schmidt-Hoberg, and F. Staub, J. High Energy Phys. 08 (2012) 074.

[15] T. Gherghetta, B. von Harling, A. D. Medina, and M. A. Schmidt, J. High Energy Phys. 02 (2013) 032.

[16] M. Perelstein and B. Shakya, Phys. Rev. D 88, 075003 (2013).

[17] D. Kim, P. Athron, C. Balazs, B. Farmer, and E. Hutchison, Phys. Rev. D 90, 055008 (2014).

[18] A. Kaminska, G. G. Ross, K. Schmidt-Hoberg, and F. Staub, J. High Energy Phys. 06 (2014) 153.

[19] M. Y. Binjonaid and S. F. King, Phys. Rev. D 90, 055020 (2014); 90, 079903(E) (2014).
[20] P. J. Fox, A. E. Nelson, and N. Weiner, J. High Energy Phys. 08 (2002) 035.

[21] Z. Chacko, P. J. Fox, and H. Murayama, Nucl. Phys. B706, 53 (2005).

[22] L. M. Carpenter, P. J. Fox, and D. E. Kaplan, arXiv:hep-ph/ 0503093.

[23] I. Antoniadis, K. Benakli, A. Delgado, M. Quiros, and M. Tuckmantel, Nucl. Phys. B744, 156 (2006).

[24] G. D. Kribs, E. Poppitz, and N. Weiner, Phys. Rev. D 78, 055010 (2008).

[25] S. D. L. Amigo, A. E. Blechman, P. J. Fox, and E. Poppitz, J. High Energy Phys. 01 (2009) 018.

[26] K. Benakli and M. D. Goodsell, Nucl. Phys. B816, 185 (2009).

[27] K. Benakli and M. D. Goodsell, Nucl. Phys. B830, 315 (2010).

[28] K. Benakli and M. D. Goodsell, Nucl. Phys. B840, 1 (2010).

[29] K. Benakli, Fortschr. Phys. 59, 1079 (2011).

[30] S. Y. Choi, D. Choudhury, A. Freitas, J. Kalinowski, and P. M. Zerwas, Phys. Lett. B 697, 215 (2011); 698, 457(E) (2011).

[31] S. Abel and M. Goodsell, J. High Energy Phys. 06 (2011) 064.

[32] K. Benakli, M. D. Goodsell, and A.-K. Maier, Nucl. Phys. B851, 445 (2011).

[33] M. Heikinheimo, M. Kellerstein, and V. Sanz, J. High Energy Phys. 04 (2012) 043.

[34] G. D. Kribs and A. Martin, Phys. Rev. D 85, 115014 (2012).

[35] J. Kalinowski, Acta Phys. Pol. B 42, 2425 (2011).

[36] R. Davies, J. High Energy Phys. 10 (2012) 010.

[37] M. D. Goodsell, J. High Energy Phys. 01 (2013) 066.

[38] K. Benakli, M. D. Goodsell, and F. Staub, J. High Energy Phys. 06 (2013) 073.

[39] S. Abel and D. Busbridge, J. High Energy Phys. 11 (2013) 098.

[40] G. D. Kribs and A. Martin, arXiv:1308.3468.

[41] C. Csaki, J. Goodman, R. Pavesi, and Y. Shirman, Phys. Rev. D 89, 055005 (2014).

[42] D. Busbridge, arXiv:1408.4605. 
[43] K. Benakli, M. Goodsell, F. Staub, and W. Porod, Phys. Rev. D 90, 045017 (2014).

[44] S. Chakraborty, A. Datta, and S. Roy, J. High Energy Phys. 02 (2015) 124.

[45] R. Ding, T. Li, F. Staub, C. Tian, and B. Zhu, Phys. Rev. D 92, 015008 (2015).

[46] A. E. Nelson and T. S. Roy, Phys. Rev. Lett. 114, 201802 (2015).

[47] D. S. M. Alves, J. Galloway, N. Weiner, and M. McCullough, Phys. Rev. D 93, 075021 (2016).

[48] S. Chakraborty, A. Datta, K. Huitu, S. Roy, and H. Waltari, Phys. Rev. D 93, 075005 (2016).

[49] M. D. Goodsell, M. E. Krauss, T. Müller, W. Porod, and F. Staub, J. High Energy Phys. 10 (2015) 132.

[50] S. P. Martin, Phys. Rev. D 92, 035004 (2015).

[51] H. Itoyama and N. Maru, Int. J. Mod. Phys. A 27, 1250159 (2012).

[52] C. Frugiuele, T. Gregoire, P. Kumar, and E. Ponton, J. High Energy Phys. 05 (2013) 012.

[53] C. Frugiuele, T. Gregoire, P. Kumar, and E. Ponton, J. High Energy Phys. 03 (2013) 156.

[54] P. Agrawal and C. Frugiuele, J. High Energy Phys. 01 (2014) 115.

[55] E. Bertuzzo, C. Frugiuele, T. Gregoire, and E. Ponton, J. High Energy Phys. 04 (2015) 089.

[56] T. Leggett, T. Li, J. A. Maxin, D. V. Nanopoulos, and J. W. Walker, arXiv:1403.3099.

[57] T. Leggett, T. Li, J.A. Maxin, D. V. Nanopoulos, and J. W. Walker, Phys. Lett. B 740, 66 (2015).

[58] G. Du, T. Li, D. V. Nanopoulos, and S. Raza, Phys. Rev. D 92, 025038 (2015).

[59] M. A. Luty and R. Sundrum, Phys. Rev. D 65, 066004 (2002).

[60] M. Luty and R. Sundrum, Phys. Rev. D 67, 045007 (2003).
[61] H. Murayama, Y. Nomura, and D. Poland, Phys. Rev. D 77, 015005 (2008).

[62] L. M. Carpenter and J. Goodman, J. High Energy Phys. 07 (2015) 107.

[63] D. S. M. Alves, J. Galloway, M. McCullough, and N. Weiner, Phys. Rev. Lett. 115, 161801 (2015).

[64] P. Diessner, J. Kalinowski, W. Kotlarski, and D. Støckinger, J. High Energy Phys. 12 (2014) 124.

[65] P. Diessner, J. Kalinowski, W. Kotlarski, and D. Støckinger, Adv. High Energy Phys. 2015, 760729 (2015).

[66] J. R. Ellis, K. Enqvist, D. V. Nanopoulos, and F. Zwirner, Mod. Phys. Lett. A 01, 57 (1986).

[67] R. Barbieri and G. Giudice, Nucl. Phys. B306, 63 (1988).

[68] D. M. Ghilencea and G. G. Ross, Nucl. Phys. B868, 65 (2013).

[69] F. Staub, arXiv:0806.0538.

[70] F. Staub, Comput. Phys. Commun. 181, 1077 (2010).

[71] F. Staub, Comput. Phys. Commun. 182, 808 (2011).

[72] F. Staub, Comput. Phys. Commun. 184, 1792 (2013).

[73] F. Staub, (2013), Comput. Phys. Commun. 185, 1773 (2014).

[74] W. Porod, Comput. Phys. Commun. 153, 275 (2003).

[75] W. Porod and F. Staub, Comput. Phys. Commun. 183, 2458 (2012).

[76] J. L. Feng, K. T. Matchev, and T. Moroi, Phys. Rev. Lett. 84, 2322 (2000).

[77] J. L. Feng, K. T. Matchev, and T. Moroi, Phys. Rev. D 61, 075005 (2000).

[78] M. D. Goodsell, K. Nickel, and F. Staub, Eur. Phys. J. C 75, 32 (2015).

[79] M. Goodsell, K. Nickel, and F. Staub, Eur. Phys. J. C 75, 290 (2015).

[80] K. Nickel and F. Staub, J. High Energy Phys. 07 (2015) 139. 